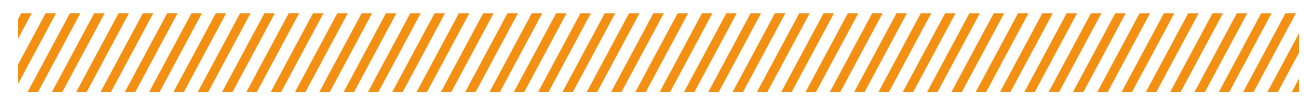

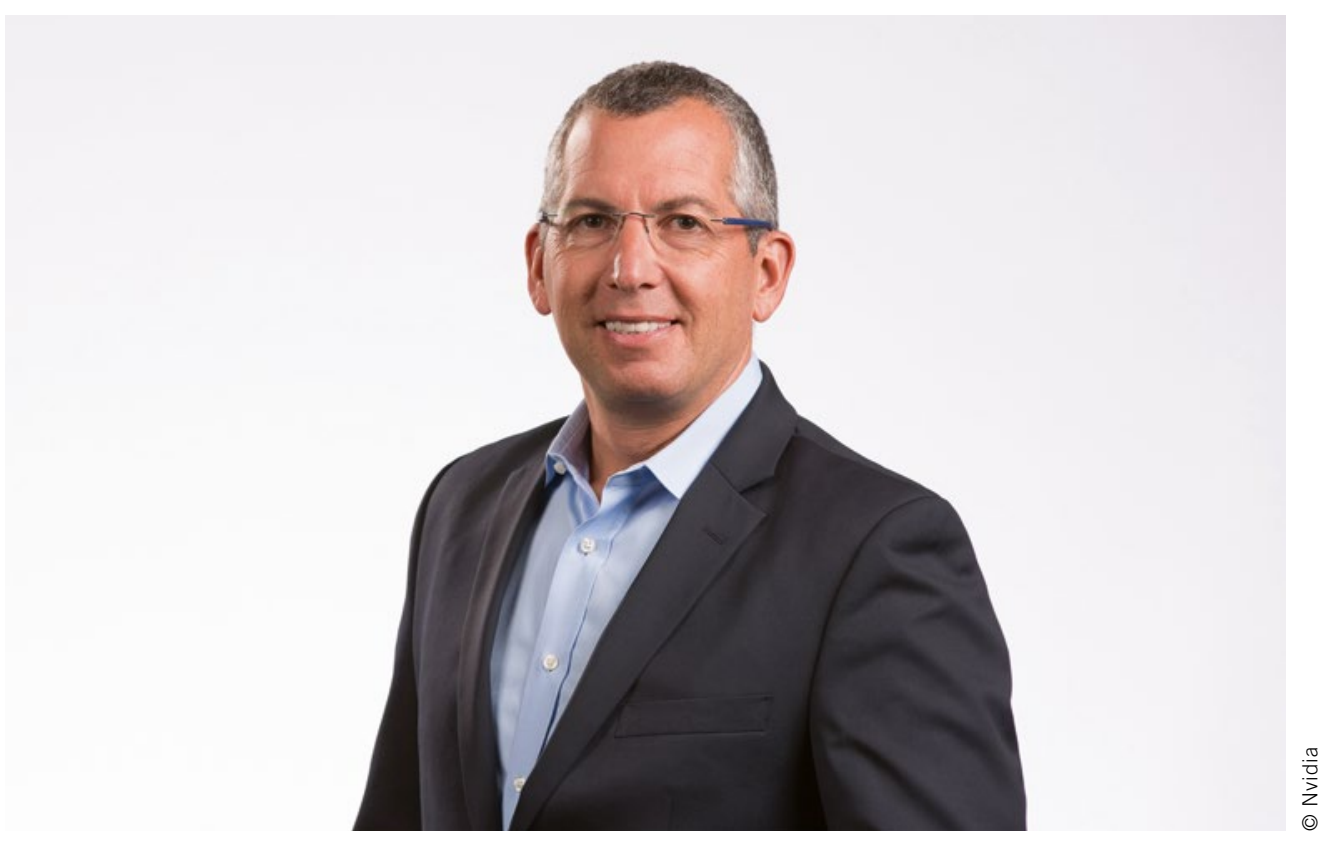

Danny Shapiro

Senior Director Automotive bei Nvidia in Silicon Valley,

Kalifornien, USA

\section{Die Zukunft der Automobilbranche ist Software-definiert}

Selbstfahrende Autos werden den Verkehr verändern und ihn sicherer und effizienter machen. Die heutigen Fahrzeugarchitekturen sind jedoch nicht in der Lage, die KI-Software, die zur Realisierung dieser Technologie benötigt wird, zu unterstützen. Die Funktionen heutiger Fahrzeuge werden von Dutzenden über das gesamte Fahrzeug verteilten elektronischen Steuergeräten realisiert. Jede ECU nimmt dabei spezialisierte Aufgaben wahr.

Anstelle von Codezeilen, die nur einer einzigen Funktion zugeordnet sind, ist KI-Software komplex und umfangreich und erfordert eine zentralisierte, leistungsstarke Recheneinheit. Mit einer solchen vereinheitlichten Architektur können OEMs fortschrittliche Softwarefunktionen integrieren und aktualisieren, sobald sie weiterentwickelt sind. Genau wie ein Mobiltelefon, das regelmäßig Software-Updates erhält, werden diese Software-definierten Fahrzeuge ständig aktualisierbar sein. Sie werden mit der Zeit immer besser.

Eine zentralisierte Fahrzeugarchitektur treibt auch die größte Veränderung des Geschäftsmodells an, welche die Automobilindustrie je erlebt hat. Durch diese neue, aufrüstbare Computingarchitektur, die auf Hochleistungscomputern basiert, können die OEMs eine loyale Kundschaft aufbauen, welche die Vorteile dieser neuen Technologie voll ausschöpfen kann. Mercedes-Benz wird zu den ersten gehören, die diese neue Architektur implementieren. Eine Software-definierte, aufrüstbare Flotte basierend auf Nvidia Drive AGX Orin geht 2024 in die Produktion.
Wenn Fahrzeugflotten von KI-Experten und Softwareingenieuren unterstützt werden, die fortschrittliche Funktionen zur kontinuierlichen Verbesserung des Fahrerlebnisses bereitstellen, können Kunden begeistert werden. Neue Funktionen, neue Fähigkeiten und neue Geschäftsmodelle gehen Hand in Hand und kommen dem Verbraucher und der Industrie zugute. Diese neuen Geschäftsmodelle werden die Beziehung zwischen OEM und Endbenutzer verändern. Dank einer Software-definierten Architektur können Autos jederzeit over-the-air mit neuen Apps und Services aktualisiert werden - für verschiedene Nutzer während der gesamten Lebensdauer des Fahrzeugs.

Die neue Architektur wird auch Entwicklern neue Möglichkeiten eröffnen. Das Nvidia-Drive-Ökosystem umfasst bereits Hunderte von OEMs, Zulieferern, Sensorikunternehmen, Kartenherstellern und Software-Startups. Mit der Fähigkeit, neue Anwendungen im Fahrzeug bereitzustellen, können mehr Entwickler beitragen und dieses Ökosystem auf noch mehr Technologien ausweiten. Und diese neuen Dienstleistungen erfordern verstärkte Unterstützungsangebote. Software, einmal erstellt, existiert ewig, und Unternehmen, die Software liefern, müssen in der Lage sein, diese zu unterstützen, solange sie genutzt wird. Nvidia setzt dieses Modell seit Jahrzehnten ein, angefangen bei unseren Gaming-Grafikkarten bis hin zu unternehmenskritischen Geschäftsanwendungen, und wird dies auch weiterhin für Fahrzeuge der Zukunft tun. 